\title{
Randomized Phase II Trial of weekly paclitaxel alone versus trastuzumab plus weekly paclitaxel as first-line therapy of patients with Her-2 positive advanced breast cancer
}

\author{
Giampietro Gasparini - Massimo Gion - Luigi Mariani - Paola Papaldo • \\ Diana Crivellari · Gianfranco Filippelli · Alessandro Morabito · \\ Vittorio Silingardi - Francesco Torino - Antonella Spada • \\ Matelda Zancan · Livia De Sio - Antonio Caputo - Francesco Cognetti · \\ Antonio Lambiase · Dino Amadori
}

Received: 14 April 2006/ Accepted: 12 June 2006/Published online: 19 July 2006

(C) Springer Science+Business Media B.V. 2006

\begin{abstract}
Background A randomized Phase II study evaluated the activity of weekly paclitaxel versus its combination with trastuzumab for treatment of patients with advanced breast cancer overexpressing HER-2.

Patients and methods Among 124 patients randomized, 123 are assessable for toxicity and 118 for response. Patients received weekly paclitaxel single agent $\left(80 \mathrm{mg} / \mathrm{m}^{2}\right)$ or combined with trastuzumab ( $4 \mathrm{mg} / \mathrm{kg}$ loading dose, then weekly $2 \mathrm{mg} / \mathrm{kg}$ ). HER-2 overexpression was determined by immunohistochemistry (IHC). Patients with $2+/ 3+$ IHC scores were eligible. IHC was compared with HER-2 serum extracellular domain (ECD).

Results Patient characteristics were similar in the two arms. Both treatments were feasible and well tolerated
\end{abstract}

G. Gasparini $(\bowtie) \cdot$ A. Morabito · F. Torino · L. De Sio Medical Oncology Division, "San Filippo Neri" Hospital, Unità Operativa Complessa di Oncologia Medica, Azienda Complesso Ospedaliero di Rilevanza Nazionale "S. Filippo Neri”, Via G. Martinotti, 20-00135 Rome, Italy

e-mail: gasparini.oncology@tiscalinet.it

M. Gion · M. Zancan

Regional Center for the Study of Biological Markers of Malignancy, General Regional Hospital, ULSS 12 Venice, Italy

L. Mariani

National Cancer Institute Milan, Unit of Medical Statistics and Biometry, Milan, Italy

P. Papaldo $\cdot$ F. Cognetti

Medical Oncology Division "A", Istituti Fisioterapici

Ospedalieri "Regina Elena", Rome, Italy with no grade 4 hematologic toxicity. No patient developed cardiac toxicity. The combined treatment was statistically significant superior for overall response rate (ORR) $(75 \%$ vs. $56.9 \% ; P=0.037)$, particularly in the subset of IHC $3+$ patients $(84.5 \%$ vs. $47.5 \% ; P=0.00050)$. A statistically significant better median time to progression was seen in the subgroup with IHC $3+(369$ vs. 272 days; $P=0.030)$ and visceral disease (301 vs. 183 days; $P=0.0080$ ) treated with combination. Multivariable analysis of predictive factors showed that only IHC score retained statistically significant value for ORR $(P=0.0035)$.

Conclusion Weekly paclitaxel plus trastuzumab is highly active and safe and it is superior to paclitaxel alone in patients with IHC score of $3+$.

Keywords Breast cancer - Trastuzumab - Paclitaxel

D. Crivellari · A. Spada

Centro di Riferimento Oncologico, Aviano, Italy

G. Filippelli · A. Caputo

Presidio Ospedaliero di Paola, Paola, Italy

V. Silingardi

Cattedra di Oncologia, Università di Modena, Modena, Italy

A. Lambiase

Hoffmann-La Roche, Milano, Italy

D. Amadori

Ospedale Morgagni - Pierantoni, Forlì, Italy 


\section{Introduction}

Innovative therapeutic strategies for breast cancer (BC) include targeted treatments against molecular pathways involved in its pathogenesis or progression $[1,2]$. Overexpression of HER-2/neu receptor and/or amplification of the HER-2 gene has been identified in $20-30 \%$ of BC and it is associated with more aggressive disease and worse prognosis [3-6]. Targeting HER-2/neu with trastuzumab, a humanized anti HER-2/neu monoclonal antibody, inhibits the growth signals mediated by this oncogene [7]. Trastuzumab demonstrated moderate activity as single agent, but showed high activity in combination with chemotherapy as front-line therapy in HER-2/neu positive BC [8-10]. In a randomized trial of first-line therapy in HER-2/neu positive BC, the combination of trastuzumab with anthracyclines or taxanes significantly improved clinical outcome [11] and quality of life [12] as compared to chemotherapy alone.

In vitro studies suggested an additive activity of trastuzumab when combined with paclitaxel $[13,14]$ and several Phase I-II clinical studies have assessed the antitumoral activity and the tolerability of such a combination [12-17]. The combination of weekly paclitaxel with trastuzumab $[10,15,16]$ produces extended cumulative exposure to the drug and ameliorates the toxicities associated with the standard tri-weekly administration [17], with mild, non-cumulative, hematologic toxicity allowing its administration for prolonged periods of time [18].

Moreover, preliminary results of a Phase III study comparing trastuzumab with weekly paclitaxel vs the tri-weekly schedule showed the superiority of the weekly administration in terms of overall response rate (ORR) and time to progression (TTP) [19].

On these basis we conducted a randomized Phase II trial to demonstrate whether trastuzumab combined with weekly paclitaxel produces superior activity in terms of ORR, TTP and duration of response as compared to weekly paclitaxel alone as first-line therapy of HER-2 overexpressing (HercepTest, score 2+ or $3+)$ BC. We also studied the correlation of the circulating levels of external cellular domain of HER-2, Epidermal Growth Factor Receptor (EGFR) and CA15-3 with clinical outcome.

\section{Patients and methods}

\section{Eligibility criteria}

Eligible patients were women with untreated metastatic BC over-expressing HER-2/neu by the Hercep-
Test assay (score $2+$ or $3+$ ) with measurable disease, age $\geq 18$ years, performance status ( 2 according to the Eastern Cooperative Oncology Group (ECOG) scale, life expectancy $>3$ months and adequate organ functions, defined as follows: Isotopic Left Ventricular Ejection Fraction (LVEF) $>50 \%$ or within normal limits (WNL) at a given Institution, AST and ALT levels $(2.5$ times the normal value, total bilirubin $<1.5$ the normal value, serum creatinine levels $(1.5 \mathrm{mg} / \mathrm{dl}$, neutrophils $\left(2,000 / \mathrm{mm}^{3}\right.$, platelets $\left(100,000 / \mathrm{mm}^{3}\right.$, hemoglobin $>10 \mathrm{~g} / \mathrm{dl}$. Patients may have received anthracycline and/or taxane containing regimens as adjuvant chemotherapy and relapsed $>12$ months following the end of chemotherapy. Patients were excluded if they had received previous chemotherapy for metastatic disease, brain, or bone metastases as the only site of disease, a history of other cancers, except in situ cervix cancer radically resected and nonmelanoma skin cancer, prior history of cardiac disease or hypertension. Patients with peripheral neuropathy of grade 2 or pregnancy were also ineligible. The protocol was approved by the Institutional Ethical Committees. Written patient informed consent was required.

Study design, randomization procedure and assignment to treatment group

This was an open-label, multicentre, randomized, phase II study. The patients were allocated by the independent monitoring agency to treatment group by randomization code envelopes, with a 1:1 randomization (paclitaxel, arm A vs. paclitaxel plus trastuzumab, arm B), stratified according to visceral involvement (yes vs. not), HER-2/neu over-expression (2+ vs. 3+) and center.

\section{Treatment plan}

Trastuzumab was administered at the loading dose of $4 \mathrm{mg} / \mathrm{kg}$ on day 1 , as 90 -min infusion followed by weekly doses of $2 \mathrm{mg} / \mathrm{kg}$ given over $30 \mathrm{~min}$. Paclitaxel was administered at the dose of $80 \mathrm{mg} / \mathrm{m}^{2}$ in $500 \mathrm{ml}$ of $0.9 \%$ normal saline over $60 \mathrm{~min}$, following trastuzumab administration or as single agent every week. Standard premedication for paclitaxel was administered. Treatment was continued until progressive disease or unacceptable toxicity.

Instructions for dose modification of paclitaxel were as follows: in presence of neutrophils $\left(1,000 / \mathrm{mm}^{3}\right.$ and platelets $\left(75,000 / \mathrm{mm}^{3}\right.$, a full dose of paclitaxel was administered. If neutrophils were $<1,000 / \mathrm{mm}^{3}$ and $>0.5 / \mathrm{mm}^{3}$, a $75 \%$ dose of paclitaxel was administered 
with G-CSF support. If neutrophils were $<500 / \mathrm{mm}^{3}$ or platelets $<75,000 / \mathrm{mm}^{3}$, a delay of a week was planned. Patients who experienced an episode of febrile or grade 4 neutropenia, were treated with a dose reduced by $25 \%$. The dose of paclitaxel was decreased to $60 \mathrm{mg} / \mathrm{m}^{2}$ also in case of grade 2 neurological toxicity. No dose reduction of trastuzumab was planned. Trastuzumab was stopped if patients developed fever, chills, allergic reactions, or any grade 3 or 4 toxicities. After withdrawal from the trial, patients were treated at the discretion ofthe investigator to cross over allowing the patients in the paclitaxel single agent arm to receive trastuzumab.

\section{Biological correlates}

HercepTest was performed by immunohistochemistry (IHC) following the manufacturer's guidelines for HER2 protein overexpression. For negative controls, the primary antibody was replaced by normal rabbit serum (Dako Negative Control Reagent). Only membrane staining intensity and pattern were evaluated using the $0 / 3+$ score.

Serum HER-2/neu was measured by the automated two-site sandwich immunoassay HER-2/neu Bayer ADVIA Centaur ${ }^{\circledR}$ System using a direct chemiluminescent technology following the manufacturer's guidelines.

Serum EGFR was measured using the Oncogene Science EGFR Microtiter ELISA by a sandwich immunoassay with a mouse monoclonal capture antibody and an alkaline phosphatase-labeled mouse monoclonal antibody as detector. Both capture and detector reagents specifically recognize the extracellular domain of EGFR.

Serum CA15.3 was evaluated using the CA15.3 Bayer ADVIA Centaur ${ }^{\circledR}$ System, a fully automated, two-step, sandwich immunoassay following the manufacturer's guidelines.

\section{Staging and follow-up procedures}

Pre-treatment evaluation was performed within 28 days before therapy with complete history and physical examination, complete blood cell counts and serum chemistries, $\mathrm{ECD}^{\mathrm{p} 185}$, left ventricular ejection fraction (LVEF) evaluated by echocardiography or MUGA scan and tumor assessment with total body computed tomography, bone scan, skeletal bone $\mathrm{X}$-rays if indicated. During study, patients underwent clinical examination and routine biochemistry before each cycle, and complete blood cell counts weekly.
Response assessment was planned after the first 8 and 16 weeks of therapy and thereafter every 12 weeks.

Evaluation of response and toxicity

Toxicities were graded according to the Version $2 \mathrm{NCI}$ Expanded Common Toxicity Criteria. The worst degree of toxicity experienced was recorded. The WHO criteria were used for measurement of response.

Statistical methods

The primary objective of the study was to determine ORR in each treatment arm. Secondary objectives were: safety profile, TTP and duration of response. We also studied the correlation of the circulating levels of ECD HER-2, EGFR and CA15-3 with clinical outcome. A sample size of 160 patients, 80 in each group, had been originally planned, calculated in order to achieve a difference in proportions with a precision (half width of the $95 \%$ confidence level) of approximately plus/minus $15 \%$, assuming an ORR of $45 \%$ for paclitaxel alone vs. $60 \%$ of the combined treatment arm. We present here the results of an unplanned interim analysis, carried out at about 4 years from study start on 124 cases.

Patients were considered evaluable for analysis of efficacy if they had received at least one cycle ( 8 weeks) of treatment. Efficacy analyses were performed on all enrolled patients (intent-to-treat population, ITT) for TTP and on evaluable patients for ORR. Duration of response was computed as the number of days from the date the objective response first recording to the date of progression. TTP was computed as the number of days between date of the first treatment infusion and date of progression, or the last date the patient was known to be progression-free. Survival was calculated from the date of enrollment to the date of death due to any cause. Duration of response, TTP and survival were analyzed using the Kaplan-Meier method.

To identify predictive indicators of response multivariable analyses were conducted using unconditional logistic regression for ORR or Cox regression model for TTP.

The measurements of circulating biomarkers were available in 89 subjects before and during treatment. Baseline marker measurements were categorized according to predefined cut-offs [20]; on-treatment measurements were transformed into the ratio vs. baseline values and categorized according to the median value. 
The multivariable models included as predictors indicator variables coding for the treatment arm, the subgroup defined by each clinical or biomarker, and pre-selected first order interaction terms to identify potential effect modification. Biomarkers were also investigated as prognostic factors. Results from multivariable models are described by reporting stratumspecific odds-ratio and hazard ratio estimates from logistic and Cox models, respectively, together with corresponding $95 \%$ confidence limits and $P$ values for interactions. Therapeutic benefit with the combined treatment is indicated by odds-ratios above one (increased response probability) or hazard ratios below one (decreased hazard of progression).

The SAS software package (version 8.2; SAS Institute Inc., Cary, NC) was used for statistical analyses. All $\mathrm{P}$ values reported are two-sided, and were considered significant when below the 5\% threshold. The CONSORT recommendations have been adopted [21].

\section{Results}

Patient characteristics

From December 2000 to September 2004, 124 patients were enrolled onto the trial of whom 123 were assessable for efficacy and toxicity and 118 for ORR (Fig. 1). The median age of the patients in arm A (paclitaxel) was 54.27 years $($ range $=30-71)$ and 56.02 (range $=32-72$ ) for arm B (paclitaxeltrastuzumab). The ECOG performance status was $0-1$ in $95.1 \%$ and $93.7 \%$ of the patients enrolled in arm $\mathrm{A}$ and $\mathrm{B}$, respectively. Patients in post-menopause were $68.3 \%$ and $66.8 \%$ in arm $\mathrm{A}$ and $\mathrm{B}$, respectively. About $44(73.3 \%)$ of the 60 patients enrolled in arm A and $40(63.5 \%)$ of the 63 patients enrolled in arm B had IHC HER2 score of $3+$. Overall, $63.4 \%$ of the patients received prior adjuvant chemotherapy, including 34 cases $(56.7 \%$ ) (arm

Fig. 1 The CONSORT

flowchart

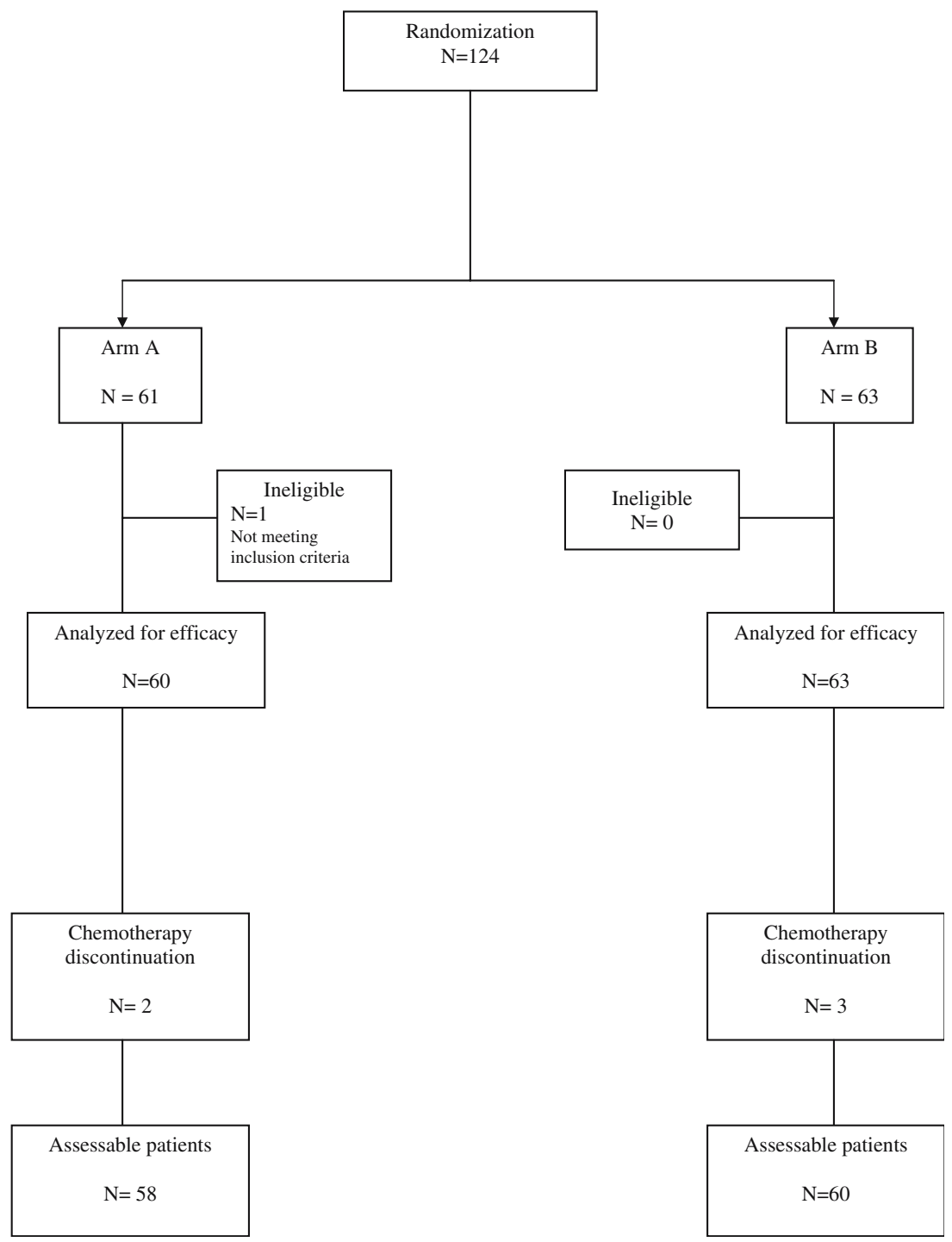


A) and 36 cases (57.1\%) (arm B) treated with anthracyclines-based regimens (Table 1).

Feasibility of study drug administration

The median number of cycles of paclitaxel per patient was 18.5 (range = 1-52) in arm A, and 21 (range = 152 ) in arm B (Table 2). The delivered dose intensity of paclitaxel was similar in both the arms: $99.31 \%( \pm 5.13)$ in arm $\mathrm{A}$ and $96.27 \%( \pm 10.57)$ in arm B, respectively. In arm B there were 1,737 trastuzumab infusions of which 1,423 in association with chemotherapy.

Safety and tolerability

A total of 123 patients were evaluable for toxicity. One patient was not evaluable because of a major protocol violation (not affected by metastatic disease).

Regarding hematologic toxicity (Table 3) no patient developed grade 4 neutropenia. Fever occurred in one patient per arm, among the 4 patients and 8 patients (arm A and arm B, respectively) who experienced grade 3 neutropenia.

Another 12 patients (arm A) and 15 patients (arm B) developed grade 1-2 neutropenia. G-CSF was administered to $10(16.7 \%)$ patients in arm $\mathrm{A}$ and to 13 $(20.6 \%)$ patients in arm B. There was no death related to sepsis or infection. No patient developed grade 3 or 4 anemia. The most frequent grade 3 or 4 non-hematologic toxicities were: alopecia (9.8\% and $17.5 \%$ in arm $\mathrm{A}$ and $\mathrm{B}$, respectively), asthenia (8.2\% and $14.2 \%$ in arm A and B, respectively), peripheral neuropathy (11.5\% and $4.8 \%$ in arm $\mathrm{A}$ and $\mathrm{B}$, respectively), and
Table 1 Patient and disease characteristics

A versus B: no statistically significant difference for each single variable

\begin{tabular}{|c|c|c|}
\hline Feature & $\begin{array}{l}\text { Paclitaxel } \\
\text { (arm A) }\end{array}$ & $\begin{array}{l}\text { paclitaxel }+ \text { trastuzumab } \\
(\operatorname{arm} B)\end{array}$ \\
\hline No. of patients & 60 & 63 \\
\hline Median age & $54.27(30-71)$ & $56.02(32-72)$ \\
\hline \multicolumn{3}{|l|}{ ECOG performance status (\%) } \\
\hline 0 & 81.7 & 80.9 \\
\hline 1 & 13.4 & 12.8 \\
\hline 2 & 4.9 & 6.3 \\
\hline \multicolumn{3}{|l|}{ Histologic type (\%) } \\
\hline Ductal & 81.7 & 84.1 \\
\hline Lobular & 10 & 6.5 \\
\hline Others & 8.3 & 9.4 \\
\hline \multicolumn{3}{|l|}{ Hormone receptor status (\%) } \\
\hline ER-/PgR- & $19(31.7 \%)$ & $31(49.2 \%)$ \\
\hline $\mathrm{ER}+/ \mathrm{PgR}+$ & $22(36.7 \%)$ & $23(36.5 \%)$ \\
\hline $\mathrm{ER}+/ \mathrm{PgR}-$ & $10(16.7 \%)$ & $3(4.8 \%)$ \\
\hline $\mathrm{ER}-/ \mathrm{PgR}+$ & $6(10.0 \%)$ & $3(4.8 \%)$ \\
\hline $\mathrm{ER}+$ /unk & $1(1.7 \%)$ & - \\
\hline Unknown & $2(3.3 \%)$ & $3(4.8 \%)$ \\
\hline \multicolumn{3}{|l|}{ Menopausal status } \\
\hline Premenopausal (\%) & $19(31.7)$ & $21(33.2)$ \\
\hline Postmenopausal (\%) & $41(68.3)$ & $42(66.8)$ \\
\hline \multicolumn{3}{|l|}{ No. of metastatic sites (\%) } \\
\hline 1 & $20(33.4)$ & $25(39.7)$ \\
\hline 2 & $24(40.0)$ & $21(33.3)$ \\
\hline 3 & $8(13.3)$ & $9(14.3)$ \\
\hline 4 & $5(8.3)$ & $5(7.9)$ \\
\hline$>4$ & $3(5.0)$ & $3(4.8)$ \\
\hline Visceral disease $(\%)$ & $43(71.7)$ & $42(66.7)$ \\
\hline Median Disease-free interval, mo (range) & $25.9(0.43-109.5)$ & $25.7(0.66-163.5)$ \\
\hline \multicolumn{3}{|c|}{ No. of patients who had adjuvant chemotherapy } \\
\hline Overall (\%) & $37(61.7)$ & $41(65.0)$ \\
\hline Anthracycline (\%) & $34(56.7)$ & $36(57.1)$ \\
\hline Taxane $(\%)$ & $3(5.0)$ & $5(7.9)$ \\
\hline $\begin{array}{l}\text { No. patients tested for HER } 2 \\
\text { overexpression }\end{array}$ & 60 & 63 \\
\hline $2+(\%)$ & $16(26.7)$ & $23(36.6)$ \\
\hline $3+(\%)$ & $44(73.3)$ & $40(63.4)$ \\
\hline Median LVEF \% (range) & $63(50-81)$ & $65(51-79)$ \\
\hline
\end{tabular}


Table 2 Study drug administration

\begin{tabular}{|c|c|c|}
\hline & $\begin{array}{l}\text { Arm A } \\
\text { (n. 60) }\end{array}$ & $\begin{array}{l}\text { Arm B } \\
\text { (n. 63) }\end{array}$ \\
\hline \multicolumn{3}{|l|}{ No. cycles } \\
\hline Total number & 1183 & $\begin{array}{l}\text { 1,423 paclitaxel } \\
1,737 \text { trastuzumab }\end{array}$ \\
\hline $\begin{array}{l}\text { Mean number/ } \\
\text { patient }\end{array}$ & $\begin{array}{l}19.72 \\
\quad( \pm 11.80)\end{array}$ & $\begin{array}{c}22.59( \pm 12.57) \\
\text { paclitaxel } \\
27.57( \pm 15.63) \\
\text { trastuzumab }\end{array}$ \\
\hline $\begin{array}{l}\text { Median number } \\
\text { (range) }\end{array}$ & $\begin{array}{l}18.50 \\
(1.0-52.0)\end{array}$ & $\begin{array}{c}21.00(1.0-52.0) \\
\text { paclitaxel } \\
25.00(1.0-55.0) \\
\text { trastuzumab }\end{array}$ \\
\hline $\begin{array}{l}\text { No. requiring dose } \\
\text { reduction }(\%)\end{array}$ & $23(1.9)$ & $\begin{array}{l}176(12.3) \text { paclitaxe } \\
0(0.0) \text { trastuzumab }\end{array}$ \\
\hline $\begin{array}{l}\text { No. of cases treated } \\
\text { with GFs }(\%)\end{array}$ & $10(16.7)$ & $13(20.6)$ \\
\hline
\end{tabular}

Table 3 Hematological toxicity

\begin{tabular}{lll}
\hline & $\begin{array}{l}\text { Arm A } \\
\text { (n. 60) No. (\%) }\end{array}$ & $\begin{array}{l}\text { Arm B } \\
\text { (n. 63) No. (\%) }\end{array}$ \\
\hline $\begin{array}{l}\text { Anemia, grade 3 } \\
\text { Neutropenia }\end{array}$ & 0 & 0 \\
Grade 3 & $4(6.6)$ & $8(12.7)$ \\
Grade 4 & 0 & 0 \\
Febbrile & $1(1.6)$ & $1(1.6)$ \\
Infection, grade 3 & $1(1.6)$ & $2(3.2)$ \\
\hline
\end{tabular}

A versus B-Pearson $\chi^{2}$ test: no statistically significant difference for each single variable

Table 4 The most frequent non-hematological toxicity

\begin{tabular}{|c|c|c|c|c|}
\hline & \multicolumn{2}{|c|}{$\begin{array}{l}\text { Arm A (n. 60) } \\
\text { No. }(\%)\end{array}$} & \multicolumn{2}{|c|}{$\begin{array}{l}\text { Arm B (n. 63) } \\
\text { No. (\%) }\end{array}$} \\
\hline & Grade 1-2 & Grade 3-4 & Grade 1-2 & Grade 3-4 \\
\hline Alopecia & $50(82.0)$ & $6(9.8)$ & $59(93.7)$ & $11(17.5)$ \\
\hline Asthenia & $38(62.3)^{* *}$ & $5(8.2)$ & $54(85.7)$ & $9(14.2)$ \\
\hline Cough & $5(8.1)$ & 0 & $8(12.7)$ & 0 \\
\hline Cutaneous rush & $5(8.2)^{*}$ & $2(3.2)$ & $15(23.8)$ & 0 \\
\hline Diarrhea & $16(26.3)$ & $1(1.6)$ & $19(30.1)$ & $2(3.2)$ \\
\hline Dyspnea & $5(8.2)$ & $1(1.6)$ & $8(12.7)$ & $2(3.2)$ \\
\hline Erythema & $5(8.2)$ & $1(1.6)$ & $9(5.3)$ & $1(1.6)$ \\
\hline Fever & $13(21.4)$ & 0 & $19(30.2)$ & 0 \\
\hline Flue & $4(6.6)$ & 0 & $8(12.7)$ & 0 \\
\hline Hepatotoxicity & $2(3.3)$ & 1 (1.6) & $4(6.6)$ & $2(3.2)$ \\
\hline Mucositis & $12(19.5)$ & 0 & $14(22.2)$ & 0 \\
\hline Nausea & $21(34.5)$ & 0 & $26(41.3)$ & 0 \\
\hline Vomiting & $13(21.4)$ & 0 & $13(21.4)$ & 0 \\
\hline Edema & $9(14.8)^{* * *}$ & $1(1.6)$ & $32(34.9)$ & $1(1.6)$ \\
\hline Onychopathy & $4(6.5)^{*}$ & $2(3.3)$ & $14(22.2)$ & $5(7.9)$ \\
\hline $\begin{array}{l}\text { Peripheral } \\
\text { neuropathy }\end{array}$ & $46(74.4)^{* *}$ & $7(11.5)$ & $60(95.3)$ & $3(4.8)$ \\
\hline
\end{tabular}

Table 5 Cardiac measurements by left ventricular ejection fraction (LVEF)

\begin{tabular}{lll}
\hline & Arm A & Arm B \\
\hline $\begin{array}{ll}\text { Baseline value } \\
\text { No. patients }\end{array}$ & 63 & 55 \\
Mean (STD) & $64.9( \pm 7.2)$ & $63.6( \pm 7.1)$ \\
Median (range) & $65(51-79)$ & $63(50-81)$ \\
Week 8 & & \\
No. patients & 40 & 35 \\
Mean (STD) & $65.1( \pm 6.9)$ & $65.9( \pm 8.6)$ \\
Median & 65 & 65 \\
Week 16 & & \\
No. patients & 40 & 28 \\
Mean (STD) & $64.1( \pm 7.4)$ & $65.2( \pm 8.5)$ \\
Median & 63.9 & 63.5 \\
1st Follow up visit & & 13 \\
No. patients & 26 & $66.5( \pm 7.7)$ \\
Mean (STD) & $65.3( \pm 5.6)$ & 65.8 \\
Median & 64.5 & 5 \\
2nd Follow up visit & 17 & $56( \pm 10.8)$ \\
No. patients & $67.4( \pm 10.5)$ & 62 \\
Mean (STD) & 66 & 3 \\
Median & & $55( \pm 8.7)$ \\
3rd Follow up visit & 8 & 50 \\
No. patients & $63.2( \pm 4.6)$ & \\
Mean (STD)* & 63 & \\
Median & & \\
\hline
\end{tabular}

*Paired Student $T$ test: no statistically significant difference

diarrhea (1.6\% and $3.2 \%$ in arm A and B, respectively) (Table 4).

No patient developed symptomatic congestive heart failure. During therapy there was no significant decrease in LVEF values to below the institutional lower limits of normal (Table 5). By comparing the baseline LVEF values with the last recorded LVEF assessment (3rd follow up $=46-54$ week), a slight decrease of LVEF was observed in arm B ( $65 \%$ vs. $63 \%)$ and in arm A (63\% vs. $50 \% ; P=.14)$, but in many subjects the last LVEF assessment was at the time of disease progression. One patient in arm A experienced an acute myocardial infarction requiring hospitalization and medical therapy. She was a 63 years old woman, affected by obesity, type II diabetes and hypothyroidism. One patient in arm B, affected by extended liver disease with ascites, pleural effusion and type II diabetes, experienced an ischemic heart attack during the first infusion.

\section{Efficacy}

Oversee One hundred-eighteen patients were assessable for ORR. Six cases were not evaluable for the following reasons: one patient in arm $\mathrm{A}$ had non measurable disease, two patients in arm B refused treatment; one patient in arm B experienced a serious 
Table 6 Clinical objective responses to treatment (118 assessable cases)

\begin{tabular}{lll}
\hline & $\begin{array}{l}\text { Arm A } \\
\text { No. (\%) }\end{array}$ & $\begin{array}{l}\text { Arm B } \\
\text { No. (\%) }\end{array}$ \\
\hline No. of evaluable patients & 58 & 60 \\
Complete response & $8(13.8)$ & $13(21.7)$ \\
Partial response & $25(43.2)$ & $32(53.3)$ \\
Stable disease & $14(24.1)$ & $11(18.3)$ \\
Disease progression & $11(18.9)$ & $4(6.7)$ \\
Overall response rate* & $33(57)$ & $45(75)$ \\
\hline
\end{tabular}

* A versus $\mathrm{B}-$ Pearson $\chi^{2}$ test: $P=0.0378$

adverse event and two patients had early progressive disease in arm A.

In arm $\mathrm{A}$, among the 58 assessable patients, 8 achieved a complete response (13.8\%) and $25(43.2 \%)$ a partial response, for an ORR of 56.9\% (95\% confidence interval [C.I. $]=43.2-69.8$ ).

In arm B, among the 60 assessable patients, 13 achieved a complete response $(21.7 \%)$ and $32(53.3 \%)$ a partial response, for an ORR of $75 \% \quad(95 \%$ C.I. $=62.1-85.3)$. The difference between the two arms was statistically significant (Pearson chi square test, $P=0.0378)$.

Another 14 patients in arm A and 11 patients in arm B had stable disease, for an overall disease control of $81 \%$ and $93.4 \%$, respectively (Table 6). In subset analysis, there was a statistically significant difference in ORR between the two arms among the patients with HER2 IHC 3+: arm B $84.5 \%(95 \%$ C.I. $=73.1 \%$ $-95.8 \%)$ vs. $47.5 \%(95 \%$ C.I. $=32.3 \%-62.6 \%)$ in arm A (Pearson $\chi^{2}$ test, $P=0.00050$ ).

The patients without visceral disease had a nonsignificant better ORR as compared to those with visceral involvement in both the arms: $74.3 \%$ vs. $57.1 \%$ for patients with visceral disease in arm B and in arm A $(P=0.19)$ respectively.

Median duration of response was 280 days and 362 days in arm A and B, respectively. Median TTP was 204 days (arm A) versus 301 days (arm B); logRank test, $P=0.076$ (Fig. 2A). Among the patients with HER2 IHC 3+ a statistically significant difference between the two groups of treatment emerged (arm A: 272 days vs. arm B: 369 days; log-Rank test, $P=0.030$ ) (Fig. 2B). Similarly, among the patients with visceral involvement there was a statistically significant difference in favour of the combined treatment (arm A: 183 days vs. 301 days arm B; log-Rank test, $P=0.0080)$.

The median follow-up was 16.6 months. The median OS has not yet been reached (because only 42 patients died).
The search for predictive factors of ORR (Table 7) yielded statistically significant results for HER2 score $(P=0.0035)$ only. An increased odds of response (OR) in arm B versus arm A occurred in the HER2 score 3+ subset $(\mathrm{OR}=5.677)$. A borderline $P$ value was observed for the number of metastatic sites $(P=0.0669)$. The benefit from the combined treatment was evident in patients with $1-2$ affected sites $(\mathrm{OR}=3.669)$, but not in case of more diffuse disease.

The average on-treatment versus baseline levels ratios for biomarkers were 0.80 for circulating HER2 and 0.85 for CA15.3 and were significantly $<1$ both in arm A $(P<0.0001$ and 0.0003 , respectively) and arm $\mathrm{B}(P=0.0029$ and 0.0103 , respectively), suggesting an effect, independent of treatment arm.

The search for predictive factors of TTP (Table 7) failed to yield significant results for all the investigated variables. However, a low $P$ value was obtained for HER2 score $(P=0.0938)$, and in the IHC $3+$ subset the upper $95 \%$ confidence limit for the hazard ratio was below one $(\mathrm{HR}=0.497,95 \%$ confidence limits: 0.260-0.952). Borderline $P$ values were obtained for baseline circulating HER2 $(P=0.0538)$ and ontreatment CA15.3 $(P=0.0666)$.

Finally, a worse prognostic effect was significantly associated with elevated baseline CA15.3 levels $(\mathrm{HR}=2.030, P=0.0217)$.

\section{Discussion}

Trastuzumab, an anti-HER2 humanized monoclonal antibody is the first molecular targeting agent with documented activity in advanced breast cancer [22].

The best clinical results have been obtained using trastuzumab in combination with chemotherapy as front-line therapy [23] and based on the results of the Phase III trial by Slamon et al. [11], FDA approved trastuzumab in combination with a standard schedule of paclitaxel.

In such a study the results from the 3-week paclitaxel plus trastuzumab subset, indicated a survival benefit compared with paclitaxel alone, although the difference between the two arms was not statistically significant (22.1 vs. 18.4 months; $P=0.17$ ).

Possible strategies to improve clinical results of the combinations of trastuzumab with taxanes include : (i) the identification of the taxane of choice; (ii) evaluation of triplets with taxanes and platinum salts or liposomal doxorubicin and (iii) the use of weekly schedules of paclitaxel or docetaxel. 

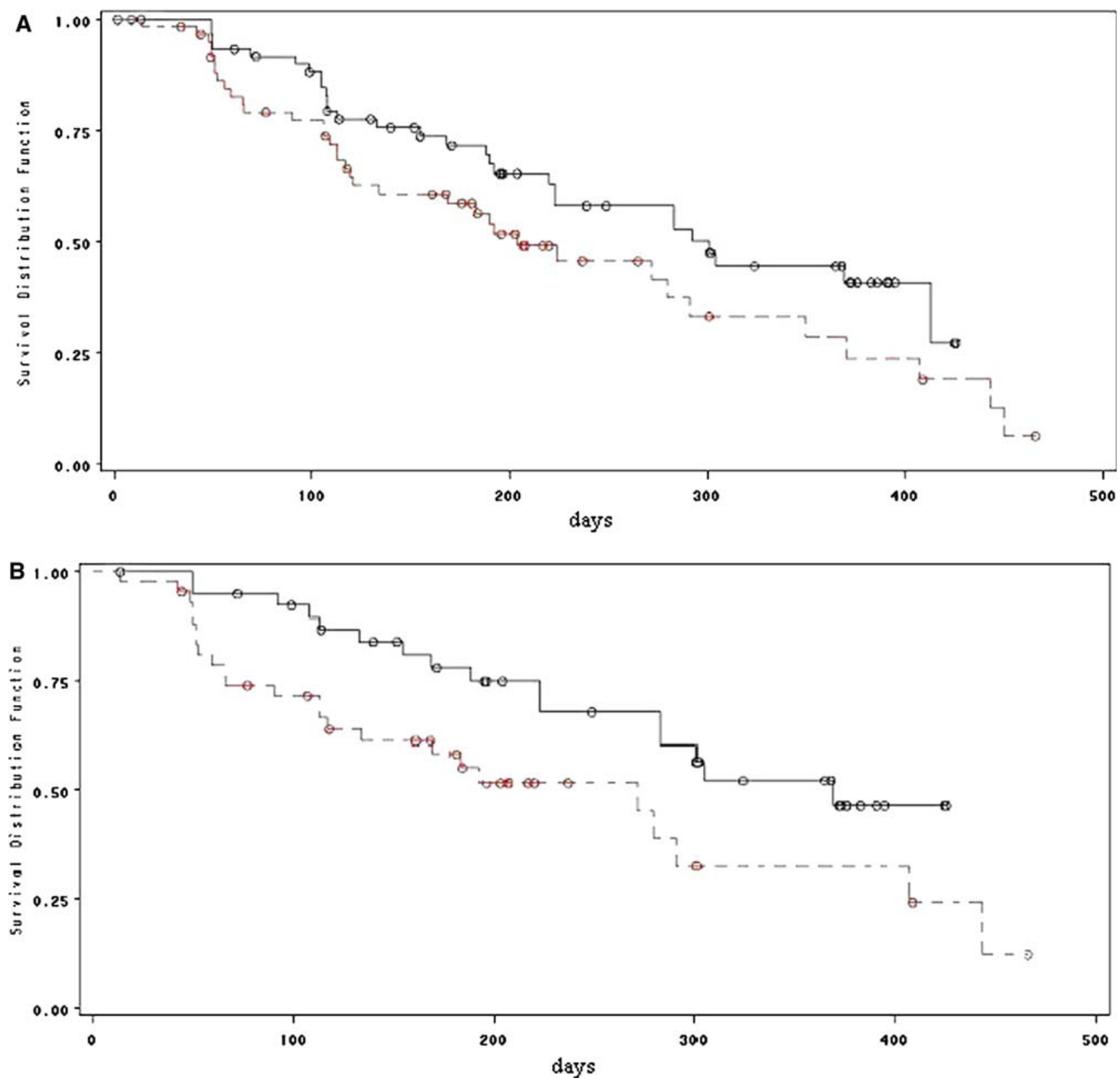

Fig. 2 (A) Overall estimated time to progression of paclitaxelalone (discontinuous line) and trastuzumab plus paclitaxel (continuous line) arms (Kaplan-Meier plots) $(P=0.076)$. (B)

As far as the first point is concerned, Jones et al. [24] in a Phase III trial of standard schedule of paclitaxel versus docetaxel showed superiority for docetaxel in all clinical endpoints.

A randomized Phase II trial by Marty et al. [25] documented that the combination of trastuzumab and standard 3-week docetaxel is significantly more active than docetaxel alone.

Pegram et al. [26] reported the results of two phase II studies (BCIRG101 and UCLA-ORN) that evalu-
Estimated time to progression of paclitaxel-alone (discontinuous line) and trastuzumab plus paclitaxel (continuous line) arms in HER2 $3+$ patients (Kaplan-Meier plots) $(P=0.03)$

ated triplets of trastuzumab, docetaxel and cisplatin or carboplatin. In both the studies an interesting response rate was obtained (ORR:79\% BCIRG101; ORR:58\% UCLA-ORN, respectively). Phase II studies, exploring triplets of trastuzumab, taxanes, and liposomal doxorubicin are ongoing, and Baselga et al. [27] reported a high overall response rate, with half of the patients with objective complete response.

Seidman et al. first explored the use of weekly schedules of paclitaxel alone [17] or combined with 
Table 7 Multivariable analysis of predictive factors for overall objective response and for time-to-progression

\begin{tabular}{|c|c|c|c|c|c|c|c|c|}
\hline \multirow[t]{2}{*}{ Stratum } & \multicolumn{4}{|c|}{ Overall objective response } & \multicolumn{4}{|l|}{ Time-to-progression } \\
\hline & Odds ratio estimate & $\begin{array}{l}95 \% \\
\text { confide } \\
\text { limits }\end{array}$ & $\begin{array}{l}\text { Wald } \\
\text { nce }\end{array}$ & $\mathrm{P}$ for interaction & Odds ratio estimate & $\begin{array}{l}95 \% \\
\text { confide } \\
\text { limits }\end{array}$ & $\begin{array}{l}\text { Wald } \\
\text { nce }\end{array}$ & $P$ for interaction \\
\hline \multicolumn{9}{|l|}{ Her2 score } \\
\hline $3+$ & 5.677 & 1.967 & 16.381 & 0.0035 & 0.497 & 0.260 & 0.952 & 0.0938 \\
\hline $2+$ & 0.329 & 0.068 & 1.590 & & 1.220 & 0.534 & 2.785 & \\
\hline \multicolumn{9}{|c|}{ Dominant site } \\
\hline Non-visceral & 2.537 & 0.613 & 10.511 & 0.8639 & 1.232 & 0.463 & 3.271 & 0.7845 \\
\hline Visceral & 2.186 & 0.845 & 5.653 & & 0.557 & 0.304 & 1.023 & \\
\hline \multicolumn{9}{|c|}{$E R / P g R$ status } \\
\hline Positive & 1.385 & 0.401 & 4.784 & 0.3079 & 0.640 & 0.289 & 1.417 & 0.7845 \\
\hline Negative & 3.282 & 1.125 & 9.573 & & 0.739 & 0.382 & 1.431 & \\
\hline \multicolumn{9}{|l|}{ Lesions (No) } \\
\hline$l-2$ & 3.669 & 1.402 & 9.604 & 0.0669 & 0.655 & 0.352 & 1.218 & 0.7355 \\
\hline$>2$ & 0.698 & 0.157 & 3.101 & & 0.792 & 0.323 & 1.939 & \\
\hline \multicolumn{9}{|l|}{$E C O G P S$} \\
\hline 0 & 2.967 & 1.224 & 7.193 & 0.1688 & 0.631 & 0.357 & 1.118 & 0.4643 \\
\hline$>2$ & 0.693 & 0.106 & 4.521 & & 1.009 & 0.334 & 3.053 & \\
\hline \multicolumn{9}{|c|}{ Baseline circulating Her2 } \\
\hline$<15$ & 2.045 & 0.464 & 9.033 & 0.6044 & 1.497 & 0.570 & 3.933 & 0.0538 \\
\hline$\geq 15$ & 1.250 & 0.406 & 3.848 & & 0.468 & 0.231 & 0.948 & \\
\hline \multicolumn{9}{|c|}{ Baseline circulating EGFR } \\
\hline$<52$ & 2.000 & 0.548 & 7.301 & 0.5996 & 0.700 & 0.308 & 1.591 & 0.9607 \\
\hline$\geq 15$ & 1.231 & 0.347 & 4.369 & & 0.720 & 0.339 & 1.527 & \\
\hline \multicolumn{9}{|c|}{ Baseline CA 15.3} \\
\hline$<31$ & 3.091 & 0.482 & 19.837 & 0.3415 & 1.408 & 0.504 & 3.934 & 0.1209 \\
\hline$\geq 31$ & 1.091 & 0.373 & 3.187 & & 0.537 & 0.271 & 1.065 & \\
\hline \multicolumn{9}{|l|}{ Her2 delta } \\
\hline$<0.80$ & 1.495 & 0.430 & 5.191 & 0.983 & 0.520 & 0.232 & 1.166 & 0.2684 \\
\hline$\geq 0.80$ & 1.524 & 0.424 & 5.473 & & 0.970 & 0.447 & 2.104 & \\
\hline \multicolumn{9}{|l|}{ EGFR delta } \\
\hline$<1.00$ & 1.339 & 0.405 & 4.426 & 0.8283 & 0.499 & 0.224 & 1.108 & 0.1984 \\
\hline$\geq 1.00$ & 1.636 & 0.421 & 6.359 & & 1.045 & 0.466 & 2.343 & \\
\hline \multicolumn{9}{|l|}{ CA153 delta } \\
\hline$<0.85$ & 1.846 & 0.515 & 6.615 & 0.6524 & 0.411 & 0.177 & 0.951 & 0.0666 \\
\hline$\geq 0.85$ & 1.224 & 0.351 & 4.269 & & 1.167 & 0.549 & 2.479 & \\
\hline
\end{tabular}

trastuzumab [10]. The results of these studies were encouraging in terms of activity and, particularly, regarding the toxicity profile.

Two additional Phase II studies by Gori et al. [15] and Fountzilas et al. [16] of trastuzumab with weekly paclitaxel, confirmed the results by Seidman et al. [10].

On the contrary, weekly docetaxel in combination with trastuzumab seems to be more toxic, with two independent studies reporting that approximately one quarter of cases were withdrawn because of severe toxicity $[28,29]$.

A major advantage of use of trastuzumab is the prolonged duration of response, therefore, it is crucial to combine it with tolerable schedules of chemotherapy and to limit the risk of cardiac toxicity, allowing for long-lasting treatments in responsive patients. Weekly paclitaxel seems to be a good option also because preclinical studies suggested that its low and frequent dosage enhances the antiangiogenic and pro-apoptotic effects [30].

We performed the present study to explore the benefit of the interaction of trastuzumab and weekly paclitaxel as front-line therapy in women with HER2 overexpressing tumors.

At the interim analysis performed after the first 124 cases enrolled we decided for ethical reasons to interrupt the accrual of patients. The decision was based both on data from other trials suggesting that only the patients with strong HER-2 overexpression (IHC score 3+) and/or gene amplification gain benefit from trastuzumab [31] and on our own results that confirmed a statistically significant superior outcome for the patients with HercepTest score 3+ and because the planned statistical difference of $15 \%$ for ORR between the two treatment arms was reached. 
We observed a high compliance to treatment with a delivered dose intensity $>96 \%$ in both the arms. No patient experienced grade 4 hematologic toxicity, $12.7 \%$ of the cases in the combination arm had grade 3 neutropenia and only one patient experienced febrile neutropenia. These results compare favourably with the data by Marty et al. [25] who reported that in the combination docetaxel-trastuzumab arm $32 \%$ of patients had grade 3-4 neutropenia with $23 \%$ of episodes of febrile neutropenia. In our study only a minority of patients had grade 3-4 non-hematologic side effects with a slightly higher incidence of asthenia $(14.3 \%$ vs. $8.2 \%$ ) and a slightly lower rate of peripheral neuropathy $(4.8 \%$ vs. $11.5 \%)$ in the combination arm. In both the arms there was no significant decrease in LVEF values. No patient had congestive heart failure, a woman treated with paclitaxel alone experienced an acute myocardial infarction resolved with medical therapy and another patient in the combination arm had a reversible episode of angina pectoris.

In both the treatment arms high ORRs were observed. The combined treatment was statistically significant more active than paclitaxel monotherapy $(75 \%$ vs. $56.9 \%$, respectively; $P=0.037$ ), with only 4 out of the $60(6.6 \%)$ assessable patients experiencing disease progression.

Subset analysis showed that the combined therapy was associated with significantly higher ORR as compared to paclitaxel monotherapy in patients with IHC score $3+$.

Also the median TTP favoured the combined therapy, but the difference did not reach a statistically significant level (204 days vs. 301 days, respectively; $P=0.076$ ). A statistically significant better median TTP for the patients treated with trastuzumab and paclitaxel was observed in the subsets of patients with HER2 IHC $3+(P=0.030)$ and visceral involvement $(P=0.0080)$. Multivariable analysis of predictive factors of ORR documented that HER2 score was the only significant predictor $(P=0.0035)$. A borderline $P$ value was retained by the number of metastatic sites $(P=0.066)$.

Circulating ECD-HER2 basal levels were significantly associated with IHC score in agreement with the study by Fornier et al. [32], but they did not retain predictive value. Similarly both circulating EGFR and CA15.3 were not predictive for response. No factor was predictive for TTP.

Finally, a significant worse prognostic effect in both the arms was associated with elevated baseline CA15.3 levels $(P=0.021)$.

Overall, trastuzumab combined with weekly paclitaxel is a highly active and safe regimen. The side effects of the schedule are manageable with a minority of patients requiring bone-marrow growth factor support and hospitalization for serious side effects. The regimen was not associated with cardiac toxicity.

In conclusion, the weekly regimen of trastuzumab and paclitaxel is recommended as one of the most active and safe regimens for women with BC with IHC HER2 of score $3+$.

\section{Authors' disclosures of potential conflicts of interest}

Dr. A. Lambiase is a Roche employer. All the other authors do not declare conflict of interest.

The study was supported by a grant of Roche-Italy.

List of participting Centers: F. Artioli (Unità Operativa Medicina Oncologica Ospedale di Carpi, Modena); C. Barone (Unità Operativa Oncologia Medica Policlinico Universitario Agostino Gemelli, Roma); C. Boni (Oncologia Medica Arcispedale S. Maria Nuova, Reggio Emilia); P.F. Conte (Oncologia Medica Ospedale S. Chiara, Pisa); L. Dogliotti (Oncologia Medica Ospedale S. Luigi, Orbassano - Torino); F. Ferraù (Oncologia Medica Presidio Ospedaliero San Vincenzo, Taormina - Catania); G. Francini (Oncologia Medica Policlinico Le Scotte, Siena); R. Labianca (Unità Operativa Oncologia Medica, Ospedali Riuniti, Bergamo); V. Lorusso (Istituto Oncologico di Bari); M. Marangolo (Divisione Oncologia Medica Ospedale S. Maria delle Croci, Ravenna); C.F. Pollera (Unità Operativa Oncologia Medica Ospedale Belcolle, Viterbo).

\section{References}

1. Ellis MJ, Hayes DF, Lippman ME (2000) Treatment of metastatic breast cancer. In: Harris JR, Lippman ME, Morrow $\mathrm{M}$ et al (eds) Diseases of the breast. Lippincott Williams and Wilkins, Philadelphia (PA), pp 749-798

2. Gasparini G, Longo R, Torino F et al (2005) Therapy of breast cancer with molecular targeting agents. Ann Oncol 16(Suppl 4):28-36

3. Salomon DS, Brandt R, Fortunato C et al (1995) Epidermal growth factor-related peptides and their receptors in human malignancies. Critical Rev Oncol Hematol 19:183-232

4. Hill CS, Treisman R (1995): Transcriptional regulation by extracellular signals; mechanisms and specificity. Cell 80:199-211

5. Slamon DJ, Clark GM, Wong SG et al (1987) Human breast cancer: correlation of relapse and survival with amplification of the HER-2/neu oncogene. Science 235:177-182

6. Quenel N, Wafflart J, Borrichon F et al (1995) The prognostic value of c-erbB2 in primary breast carcinomas: a study of 942 cases. Breast Cancer Res Treat 35:283-291

7. McKeage K, Perry CM (2002) Trastuzumab. Drugs 62:209_ 243 
8. Pegram MD, Lipton A, Hayes DF et al (1998) Phase II study of receptor-enhanced chemosensitivity using recombinant humanized anti-p185 HER2/neu monoclonal antibody plus cisplatin in patients with HER2/neu-overexpressing metastatic breast cancer refractory to chemotherapy treatment. J Clin Oncol 16:2659-2671

9. Burstein HJ, Kuter I, Campos SM et al (2001) Clinical activity of trastuzumab and vinorelbine in women with HER2-overexpressing metastatic breast cancer. J Clin Oncol 19:2722-2730

10. Seidman AD, Fornier MN, Esteva FJ et al (2001) Weekly trastuzumab and paclitaxel for metastatic breast cancer with analysis of efficacy by HER2 immunophenotype and gene amplification. J Clin Oncol 19:2587-2595

11. Slamon DJ, Leyland-Jones B, Shak S et al (2001) Use of chemotherapy plus a monoclonal antibody against HER2 for metastatic breast cancer that overexpresses HER2. N Engl J Med 344:783-792

12. Osoba D, Slamon DJ, Burchmore M et al (2002) Effects on quality of life of combined trastuzumab and chemotherapy in women with metastatic breast cancer. J Clin Oncol 20:31063013

13. Pegram M, Hsu S, Lewis G et al (1999) Inhibitory effects of combinations of HER-2/neu antibody and chemotherapeutic agents used for treatment of human breast cancers. Oncogene 18:2241-2251

14. Pegram MD, Konecny GE, O'Callaghan C et al (2004) Rational combinations of trastuzumab with chemotherapeutic drugs used in the treatment of breast cancer. J Natl Cancer Inst 96:739-749

15. Gori S, Colozza M, Mosconi AM et al (2004) Phase II study of weekly paclitaxel and trastuzumab in anthracycline- and taxane-pretreated patients with HER2-overexpressing metastatic breast cancer. Br J Cancer 90:36-40

16. Fountzilas G, Tsavdaridis D, Kalogera-Fountzila A et al (2001) Weekly paclitaxel as first-line chemotherapy and trastuzumab in patients with advanced breast cancer. A Hellenic Cooperative Oncology Group phase II study. Ann Oncol 12:1545-1551

17. Seidman AD, Hudis CA, Albanel J et al (1998): Dose-dense therapy with weekly 1-hour paclitaxel infusions in the treatment of metastatic breast cancer. J Clin Oncol 16:33533361

18. Perez EA, Vogel CL, Irwin DH et al (2001) Multicenter phase II trial of weekly paclitaxel in women with metastatic breast cancer. J Clin Oncol 19:4216-4223

19. Seidman AD, Berry D, Cirrincione C et al: CALGB 9840 (2004) Phase III study of weekly (w) paclitaxel (P) via 1-hour(h) infusion versus standard (S) $3 \mathrm{~h}$ infusion every three week in the treatment of metastatic breast cancer (MBC), with trastuzumab (T) for HER2 positive MBC and randomized for T in HER2 normal MBC. Proc Am Soc Clin Oncol 22:512 (abstr)

20. Dittadi R, Zancan M, Perasole A et al (2001) Evaluation of HER-2/neu in serum and tissue of primary and metastatic breast cancer patients using an automated enzyme immunoassay. Int J Biol Markers 16:255-261

21. Moher D, Schulz KF, Altman DG (2001) The CONSORT statement: revised recommendations for improving the quality of reports of parallel-group randomised trials. Lancet 357:1191-1194

22. Baselga J, Tripathy D, Mendelsohn J et al (1996) Phase II study of weekly intravenous recombinant humanized antip185 HER2 monoclonal antibody in patients with HER2/ neu-overexpressing metastatic breast cancer. J Clin Oncol 14:737-744

23. Bell R, Verma S, Untch M et al (2004) Maximizing clinical benefit with trastuzumab. Semin Oncol 31(Suppl 10):35-44

24. Jones S, Erban J, Overmoyer B et al (2003) Randomized trial comparing docetaxel and paclitaxel in patients with metastatic breast cancer. Breast Cancer Res Treat 82:10(abstr)

25. Marty M, Cognetti F, Maraninchi D et al (2005) Randomized Phase II trial of the efficacy and safety of trastuzumab combined with docetaxel in patients with human epidermal growth factor receptor 2-positive metastatic breast cancer administered as first-line treatment: the M77001 Study Group. J Clin Oncol 23:4265-4274

26. Pegram MD, Pienkowski T, Northfelt DW et al (2004) Results of two open-label, multicenter phase II studies of docetaxel, platinum salts, and trastuzumab in HER2-positive advanced breast cancer. J Natl Cancer Inst 96:759-769

27. Baselga J, Climent MA, Lluch A et al (2004) Results of a phase II study of liposomal doxorubicin (Myocet) in combination with weekly paclitaxel and trastuzumab (Herceptin) in patients with HER2-positive locally advanced or metastatic breast cancer (LA/MBC). Eur J Cancer 2:262 (abstr)

28. Esteva FJ, Valero V, Booser D et al (2002) Phase II study of weekly docetaxel and trastuzumab for patients with HER-2overexpressing metastatic breast cancer. J Clin Oncol 20:1800-08

29. Tedesco KL, Thor AD, Johnson DH et al (2004): Docetaxel combined with trastuzumab is an active regimen in HER-2 $3+$ overexpressing and fluorescent in situ hybridization-positive metastatic breast cancer: a multi-institutional Phase II trial. J Clin Oncol 22:1071-1077

30. Bocci G, Nicolaou KC, Kerbel RS (2002) Protracted lowdose effects on human endothelial cell proliferation and survival in vitro reveal a selective antiangiogenic window for various chemotherapeutic drugs. Cancer Res 62:6938-6943

31. Perez EA, Pusztai L, van De Vijver (2004) Improving patient care through molecular diagnostics. Sem Oncol 31(Suppl 10):14-20

32. Fornier MN, Seidman AD, Schwartz MK et al (2005) Serum HER2 extracellular domain in metastatic breast cancer patients treated with weekly trastuzumab and paclitaxel: association with HER2 status by immunohistochemistry and fluorescence in situ hybridization and with response rate. Ann Oncol 16:234-239 